\section{Sind Laserdrucker gesundheitsschädlich?}

— In Fallbeschreibungen wird immer wieder über gesundheitliche Beschwerden wie Augenreizungen, Atemwegsprobleme oder Kopfschmerzen berichtet, die beim Arbeiten im Umfeld von Laserdruckern auftreten. Ein Kausalzusammenhang sei allerdings oft nicht belegt, sagte PD Dr. Rudolf A. Jörres, Ludwig-Maximilians-Universität München. Bekannt ist zwar, dass Laserdrucker je nach Modell Nanopartikel in unterschiedlicher Menge ausstoßen, die gesundheitliche Relevanz solcher Emissionen wird jedoch kontrovers diskutiert.

In-vitro-Untersuchungen mit verschiedenen Zell-Linien - Atemwegsepithelzellen, Makrophagen und Lymphoblasten - weisen beispielsweise darauf hin, dass Emissionen aus Laserdruckern ungünstige Auswirkungen auf die Atemwege haben können: Die Partikelbelastung führte zu Schädigungen der Zellmembran, einer erhöhten Bildung Reaktiver Sauerstoff-Spezies (ROS) sowie einer vermehrten Freisetzung proinflammatorischer Zytokine [Pirela SV et al. Environ Health Perspect 2015 (Epub ahead of print)].
Solche Untersuchungen seien zwar aufschlussreich, aber nur bedingt auf den Menschen übertragbar, so Jörres. So fanden sich beispielsweise in einer Expositionsstudie der LMU München und der Bundesanstalt für Materialforschung und -prüfung keine Belege dafür, dass Emissionen aus Laserdruckern messbare Gesundheitsschäden hervorrufen.

Teilnehmer der Studie waren 23 gesunde Kontrollpersonen sowie 14 Probanden mit leichtem Asthma und 15 Betroffene mit anamnestischen Beschwerden beim Umgang mit Laserdruckern. Die Probanden wurden unter standardisierten Bedingungen über jeweils 75 Minuten sowohl extrem hohen als auch sehr niedrigen Emissionen ausgesetzt. Um mögliche respiratorische Effekte zu überprüfen, wurden vor und nach der Exposition unter anderem verschiedene Lungenfunktionsmessungen durchgeführt sowie Zytokine in Nasensekret und Serum bestimmt. Die Auswirkungen der Druckeremissionen waren gering bis sehr gering - auch nach den

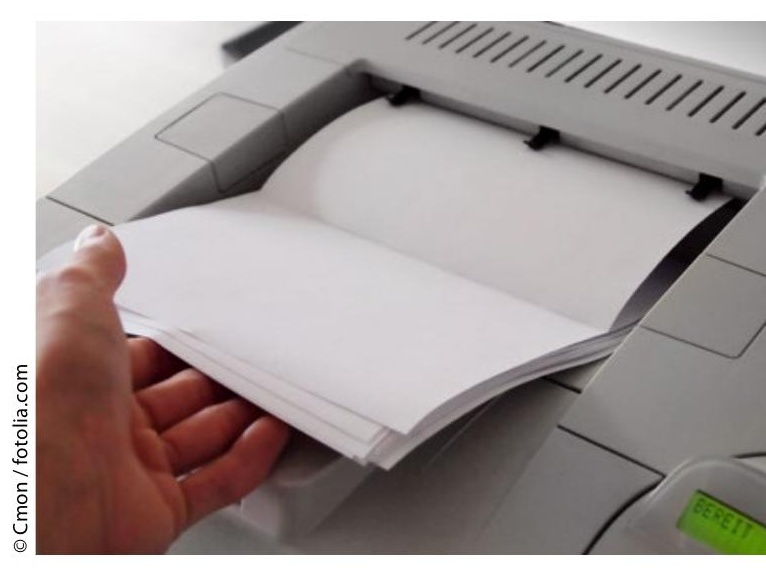

extrem hohen Expositionsleveln und obwohl Personen eingeschlossen waren, die als besonders sensitiv gelten können, so Jörres.

Es sei zwar denkbar, dass Emissionen aus Laserdruckern eine gesundheitsschädliche Potenz haben, fasste Jörres die derzeitige Datenlage zusammen, klinische Evidenz gibt es dafür allerdings bislang nicht.

Angelika Bauer-Delto

AllergoConnect: Klimawandel, Stadtplanung und Allergieprävention

\section{Chronisch spontane Urtikaria: In-label geht vor off-label}

— Die Zulassung des monoklonalen antiIgE-Antikörpers Omalizumab hat Patienten mit chronisch spontaner Urtikaria (CSU) zu einer besseren Lebensqualität verholfen und mit der Erkrankung befassten Dermatologen gleichzeitig zu einem besseren Krankheitsverständnis. Kaum eine andere Erkrankung aus dem dermatologisch-allergologischen Umfeld verdiene so dringlich ein Update wie die CSU, befand Prof. Dr. Marcus Maurer, Berlin.

Beim Bemühen, die Patienten symptomfrei zu bekommen, ist die Verordnung eines Antihistaminikums nach wie vor die erste Option - falls nötig auch mit einer bis zu vierfach erhöhten Standarddosis. Mit dem Hinweis auf deutlich erhöhte Unfallraten erteilte Maurer der Verordnung sedierender Antihistaminika eine klare Absage. Stellt sich unter hoch dosierter Antihistaminikagabe der gewünschte Behandlungserfolg nicht ein, rechtfertigen die von diversen Fachgesellschaften propagierten Therapieemp- fehlungen bereits einen Behandlungsversuch mit Omalizumab - und zwar noch vor der Gabe nicht zugelassener Medikamente wie zum Beispiel Cyclosporin A: „Sie tun sich keinen Gefallen, wenn Sie nicht zugelassenen Medikamenten den Vorzug vor zugelassenen geben", verwies Maurer auf eine Stellungnahme der Krankenkassen. Demnach gelte ganz klar: „In-Label vor off-Label.“ Die Ergebnisse einiger klinischer Studien (ASTERIA, GLACIAL, X-ACT) hat Maurer als Beleg dafür ins Feld geführt, dass der monoklonale anti-IgE-Antikörper Omalizumab die medikamentöse Therapie von CSU-Patienten wesentlich bereichert hat. Unter anderem nimmt unter dieser Therapie nicht allein die Zahl der Angioödemattacken ab, sondern auch die Größe der Schwellungen. Die Tatsache, dass ein Teil der Patienten bereits nach der ersten subkutanen Injektion auf die Behandlung anspricht, ein Teil jedoch erst nach wiederholten Injektionen, weist nach Einschätzung Maurers auf unterschiedliche
Wirkmechanismen des Anti-IgE-Antikörpers hin. Entschließt man sich bei CSU-Patienten zu einer Omalizumab-Behandlung, sollte diese in keinem Falle nach einem ersten Zyklus abgebrochen werden. Mit Spannung erwartet werden derzeit die Ergebnisse laufender Studien mit höher potenten AntiIgE-Antikörpern wie Ligelizumab.

Dr. Ludger Riem

AllergoCompact: Dermatologie - Allergologie aktuell (Update Urtikaria)

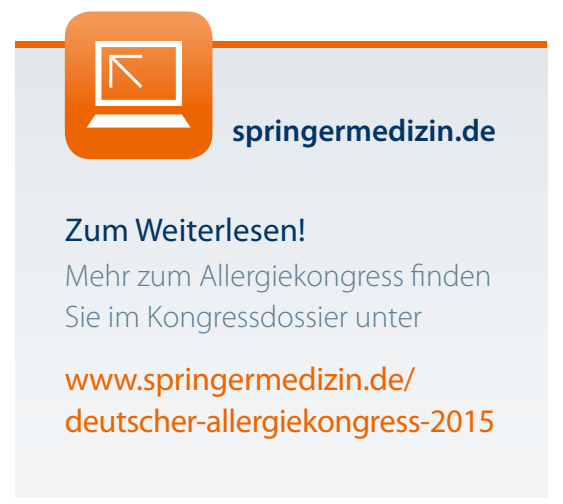

